Accepted Manuscript

Pattern Recognition for High Performance Imaging

Xiao Bai, Jun Zhou, Antonio Robles-Kelly

PII:

S0031-3203(18)30155-9

DOI:

10.1016/j.patcog.2018.04.021

Reference:

PR 6535

To appear in:

Pattern Recognition

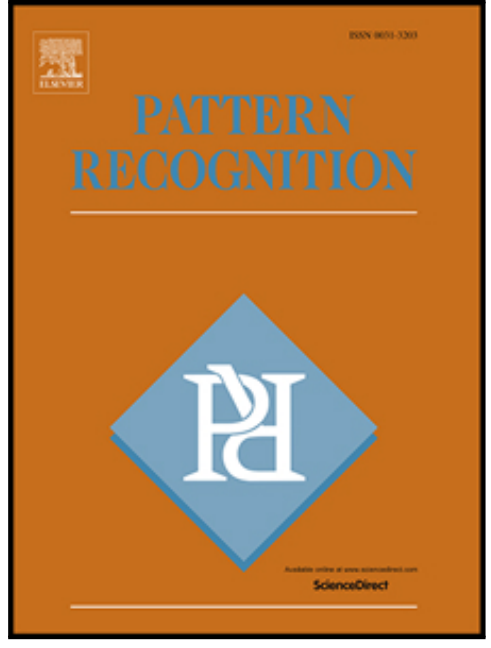

Please cite this article as: Xiao Bai , Jun Zhou, Antonio Robles-Kelly, Pattern Recognition for High Performance Imaging, Pattern Recognition (2018), doi: 10.1016/j.patcog.2018.04.021

This is a PDF file of an unedited manuscript that has been accepted for publication. As a service to our customers we are providing this early version of the manuscript. The manuscript will undergo copyediting, typesetting, and review of the resulting proof before it is published in its final form. Please note that during the production process errors may be discovered which could affect the content, and all legal disclaimers that apply to the journal pertain. 


\section{Pattern Recognition for High Performance Imaging}

\section{1、Introduction}

High performance imaging technology generates images with high spectral and spatial resolution, high dynamic range, and/or at high speed. Hyperspectral images contain tens or hundreds of contiguous wavelength indexed bands that are related to material information. High spatial resolution images provide fine details on target objects. High dynamic range images present a great range of luminance levels to capture vivid lights or shadows. High speed cameras offer high frame rate to record fast moving objects. Sometimes, high performance imaging can also be achieved by combing the output of a large number of imaging devices.

While high performance imaging has greatly expanded the sensing capability of cameras to capture scenes or phenomena that are beyond human vision, the processing, analysis and understanding of these imaging modalities are still challenging, with many unsolved problems. In particular, various types of high performance images have their unique properties, and are normally in very larger size. As a consequence, though the state-of-the-art pattern recognition techniques have achieved great success on traditional grayscale and colour images, for example, in object detection and image classification, they cannot be directly applied to high performance images. On the other hand, this also brings new opportunities to the research community, as there are strong needs to develop effective and efficient methods for a variety of pattern recognition tasks on these images.

The goal of this special issue is to provide a forum for researchers and practitioners in the broad computer vision and pattern recognition community to present their novel and original pattern recognition research for high performance imaging. We hope this special issue will become an enlightening and useful source on high performance imaging research, and also for wider research community in pattern recognition.

\footnotetext{
${ }^{*}$ Corresponding author.
} 


\section{2、Overview of the accepted papers}

We have received 23 high quality submissions and accepted 8 papers after a rigorous review process. Each submission has been assigned at least three peer reviewers and has gone through at least two rounds of revisions. The accepted papers cover some key tasks in pattern recognition, such as preprocessing of images, depth estimation, data reconstruction, object detection and recognition, and classification. They also involve images captured by different high performance imaging systems, including hyperspectral, depth field, RGB-D, and magnetic resonance.

Hyperspectral Imaging: Among all accepted papers, four of them are on hyperspectral image processing and classification.

The article "4DCAF: A Temporal Approach for Denoising Hyperspectral Image Sequences" written by Priego et al. presents a method, the very first on the topic, for denoising of hyperspectral videos. The approach is based on spatio-spectral-temporal cellular automata-based filtering, which demonstrates several advantages and outperforms several state-of-art algorithms on both simulated and real sequences.

Salient object detection from hyperspectral images is a new research topic. In the paper "Material Based Salient Object Detection from Hyperspectral Images" written by Liang et al, spatial and spectral features are used to extract material information in an image, which in turn contribute to the detection task. The proposed method first estimate the material distribution in the scene using hyperspectral unmixing approach. This distribution is used to calculate the conspicuity maps based on their global spatial variance. Then the conspicuity maps are used for final salient object detection.

To address the hyperspectral image classification problem, Deng et al. proposes method by investigating domain adaption with active learning methods. The main idea of the paper "Active Multi-Kernel Domain Adaptation for Hyperspectral Image Classification" is to retrain the multi-kernel classifier by utilizing the available labeled samples from the source domain, and adding minimum number of the most informative samples with active queries in the target domain.

\footnotetext{
* Corresponding author.
} 
In the paper of Shi and Pun, "Superpixel-based 3D Deep Neural Networks for Hyperspectral Image Classification", a deep learning method is described to extract the 3D spectral-spatial information for hyperspectral image classification. This paper combines the idea of superpixels, which represent the structure of hyperspectral image, with 3D deep neural network to improve the classification performance, and showed promising performance in handling noisy images and boundary misclassification.

Depth Information in High Performance Imaging: Three accepted papers explore various aspects on improving or using depth information in an image.

The article "Efficient Auto-Refocusing for Light Field Camera" by Zhang et al. addresses the deficiency of current refocusing approaches which are mainly based on rendering and selection and need a large number of refocused images. The proposed method is a solution based on modeling the refocusing point spread function to significantly improve the efficiency of auto-refocusing of light-field cameras.

Sheng et al. propose a method to extract epipolar plane images in all available directions to make full use of the regular grid light field images. In the article "Occlusion-Aware Depth Estimation for Light Field Using Multi-Orientation EPIs", the authors extract epipofar plane images in all available directions to make full use of the regular grid light field images. The authors designed a novel framework to estimate the depth information, which combines the local depth with edge orientation. The multi-orientation EPIs and optimal orientation selectionare proyed to be effective in detecting and excluding occlusions.

Ren et al. investigate the problem of person re-identification in the article "Multi-Modal Uniform Deep Learning for RGB-D Person Re-identification". In this method, RGB-D images are used instead of RGB images to exploit depth information for person re-identification. A deep network is developed to extract anthropometric features from processed depth images. Features are extracted from both depth images and RGB images through the network from with a uniform latent variable, and then combined by a multi-modal fusion layer jointly optimized with two CNN networks.

\footnotetext{
* Corresponding author.
} 
High Performance MR Imaging: The final paper introduces a high performance medical data application.

Zhang et al. presents a compressed sensing method for reconstruction of MRI data in "Atlas-based reconstruction of high performance brain MR data". A probabilistic atlas is employed to control the level of gradient regularization at each image location within a weighted TV regularization prior. This method conquers the drawbacks of the standard total variation approaches which sometimes over-smooth edges in the image, causing loss of important details.

\section{Acknowledgments}

We would like to thank all the authors for their valuable contributions to this special issue. We would like also to thank all reviewers for dedicated work which guarantees the high quality of accepted papers. We would like to express our gratitude to the Editor-in-Chief, Prof. Edwin Hancock, for his encouragement on editing this special issue, and to Ms. Qian Jiao, Publishing Content Specialist from Elsevier, for her excellent support in handling this special issue.

Xiao Bai*

School of Computer Science and Engineer

Beihang University, China Email address: baixiao@buaa.edu.cn

Jun Zhou

School of Information and Communication Technology Griffith University, Australia Email address: jun.zhou@griffith.edu.au

Antonio Robles-Kelly Data61 CSIRO, Australia Email address: antonio.robles-kelly@data61.csiro.au

\footnotetext{
* Corresponding author.
} 